\title{
Rentabilidad diagnóstica de los cilindros de los cuernos laterales en las biopsias prostáticas ampliadas a 10 muestras
}

\author{
Ramírez Backhaus M, Trassierra Villa M, Arlandis Guzmán S, Bosquet Sanz M, Pontones \\ Moreno JL, Jiménez Cruz JF.
}

Servicio de Urología. Hospital Universitario La Fe. Valencia

Actas Urol Esp. 2007;31(1):11-16

\begin{abstract}
RESUMEN
RENTABILIDAD DIAGNÓSTICA DE LOS CILINDROS DE LOS CUERNOS LATERALES EN LAS BIOPSIAS PROSTÁTICAS AMPLIADAS A 10 MUESTRAS

Objetivo: Valorar si el aumento del número de cilindros en la biopsia de próstata incrementa el rendimiento diagnóstico de esta prueba.

Material y método: En marzo del 2005 iniciamos este estudio prospectivo pacientes que son sometidos a una primera biopsia de próstata por un PSA entre 4 y $10 \mathrm{ng} / \mathrm{ml}$ y tacto rectal negativo. Se les lleva a cabo una biopsia prostática transrectal ecodirigida con 10 punciones según el siguiente esquema: 6 biopsias según la técnica sextante clásica, a los que añadimos 4 cilindros de las zonas más laterales de la próstata (cuernos laterales). Se analizan las variables: Edad, PSA total, PSA libre/PSA total, volumen prostático y PSA densidad.

Resultados: Valoramos 90 pacientes consecutivos. Se diagnosticó adenocarcinoma prostático a 37 de los 90 pacientes, es decir, la tasa diagnóstica de la biopsia ampliada alcanzó el 41\%. Sin embargo, la de la sextante clásica fue del 32,2\%. Estas diferencias fueron estadísticamente significativas (test de Mcnemar 0,008); además esto supone un aumento en la rentabilidad diagnóstica del 27\%. Los cilindros "extra" en los cuernos laterales detectaron 8 tumores que no eran objetivados en los cilindros de la sextante, y 5 de ellos presentaban un Gleason mayor o igual a 6.

Conclusiones: En nuestro centro, pensamos que la biopsia ampliada a 10 cilindros es una estrategia adecuada para los pacientes con sospecha de adenocarcinoma prostático que van a ser sometidos a su primera biopsia. Teniendo en cuenta la relevancia clínica de tumores dianosticados gracias a los cilindros "extra". No creemos que esta modalidad implique un sobrediagnóstico y consecuente sobretratamiento del cáncer de próstata.
\end{abstract}

Palabras clave: Cáncer de próstata. Diagnóstico. Biopsia prostática.

\section{ABSTRACT}

YIELD DIAGNOSIS OF THE PERIPHERAL CORES IN 10 NEEDLE EXTENDED PROSTATE BIOPSY

Objective: To value if the increase in the number of cylinders in the prostate's biopsy raise the diagnostic performance of this test.

Material and Method: In March 2005 we initiate this prospective study with patients who are subject to a first prostate Biopsy by a PSA between 4 and $10 \mathrm{ng} / \mathrm{ml}$ and negative rectal touch. Transrectal, echodirected prostatic biopsies with ten punctures are carried out according to the following programme as follows: 6 Biopsies according to classic sextant technique, to what we add 4 cylinders from the most lateral zones of the prostate (lateral horns). The variables are analyzed: Age, Total PSA, Free PSA/Total PSA, prostatic volume and PSA density.

Results: We value 90 patients consecutively. Prostatic adenocarcinoma was diagnosed to 37 of the 90 patients, which means that the diagnostic rate of the extended Biopsy reached a $41 \%$. Nevertheless, the rate for the classic sextant Biopsy was $32.3 \%$. These differences are statistically significant (Mcnemar test 0,008); besides, this supposes a $27 \%$ increase in the diagnostic rentability. The "extra" cylinders in the lateral horns detected 8 tumours which were not detected in the cylinders of the the sextant, and 5 of them presented a Gleason higher or equal to 6.

Conclusions: In our centre we think that the Biopsy extended to 10 cylinders is an adequate strategy for potential prostatic adenocarcinoma patients who are going to be subject to their first Biopsy. Realizing the clinic relevance of the tumours diagnosed thanks to "extra" cylinders, we do not think that this modality implies an overdiagnosis and consequently an overtreatment of the prostate cancer.

Keywords: Prostate cancer. Diagnosis. Prostate biopsy. 
$\mathrm{E}$ n su comienzo la biopsia de próstata se realizaba a ciegas sobre las zonas sospechosas prostáticas según el tacto rectal. En los años 80 con el creciente interés por el cáncer de próstata y la determinación del antígeno prostático específico (PSA) ${ }^{1}$ como marcador tumoral, la ecografía transrectal se convierte en una técnica imprescindible para la biopsia de próstata. Se desarrollan desde entonces con mucha rapidez los transductores de alta frecuencia, que permiten identificar determinadas características del cáncer de próstata $^{2}$ y los dispositivos de resorte, que facilitan la obtención del tejido prostático ${ }^{3}$.

En 1989 Torp-Pedersen et $\mathrm{al}^{2}$ introducen la biopsia de próstata ecodirigida como técnica diagnóstica en el cáncer de próstata. Aparecen los primeros estudios que demostraban la superioridad de la biopsia ecodirigida frente a la digitodirigida $^{4}$, ya que aumentaba la sensibilidad y facilitaba la punción de las zonas sospechosas en la escala de grises. Más tarde y con el fin de identificar mejor los tumores clínicamente silentes surge la sistematización de la biopsia con toma de seis muestras por punción convirtiéndose en la referencia de todas las nuevas modalidades de muestreo prostático, cuyo objetivo es incluir las áreas donde con mayor frecuencia se desarrolla el cáncer.

Hasta que se demuestre el valor de los contrastes sonográficos o la ecografia tridimensional ${ }^{5-9}$, la sistematización topográfica de la biopsia de próstata es imprescindible, pues si bien las áreas sospechosas en la escala de grises tienen dos veces más probabilidad de ser un cáncer que las isoecoicas $^{10-12}$, la mayoría de ellas siguen sin ser cáncer y el 50\% de los cánceres no palpables, con un diámetro menor de $1 \mathrm{~cm}$, no se objetivan en la ecografia ${ }^{8}$. Entre el 25 y el $50 \%$ de los cánceres pasaría inadvertido si únicamente se biopsiarán las áreas hipoecoicas ${ }^{13}$.

La rentabilidad diagnóstica de la biopsia prostática ecodirigida con toma sistemática de seis cilindros según la descripción realizada por Hodge $^{4}$, tiene un rendimiento que varía en función de las características de los pacientes a estudio. En nuestro centro con una serie histórica de 303 pacientes con un PSA medio de 11,67 y volumen prostático medio de $52,6 \mathrm{ml}$; obteníamos una tasa de detección de tumor del 26,7\%, y cuando seleccionamos los pacientes con tacto rectal negativo y PSA entre 4 y 10, la tasa diagnóstica no alcanzaba el $20 \%{ }^{14}$. Nos plantemos, por tanto, aumentar el número de cilindros a expensas de los cuernos laterales prostáticos, y valorar la rentabilidad de estos 4 nuevos cilindros.

\section{MATERIAL Y MÉTODO}

Desde marzo del 2005 hasta febrero del 2006 efectuamos 261 biopsias a 256 pacientes. Realizamos un estudio prospectivo sobre 90 pacientes que cumplen los siguientes criterios de inclusión:

1. Primera biopsia.

2. Tacto rectal negativo.

3. PSA entre 4 y 10.

Se realiza la biopsia con el ecógrafo Siemens Sonoline Antares ${ }^{\circledR}$ y un dispositivo de resorte con una aguja de punción $18 \mathrm{G}$, de $25 \mathrm{~cm}$ de longitud que obtiene cilindros de $25 \mathrm{~mm}$ y aproximadamente $1 \mathrm{~mm}$ de grosor.

Aconsejamos al paciente la aplicación de un enema de limpieza la noche antes de la biopsia y otro la mañana de la prueba. El paciente es recibido en la unidad de cirugía sin ingreso y es dado de alta a las $2 \mathrm{~h}$ de la realización de la prueba. Para el control del dolor operatorio utilizamos petidina $1 \mathrm{mg} / \mathrm{kg}$ de peso diluido en $100 \mathrm{ml} \mathrm{de}$ suero fisiológico media hora antes de practicar la biopsia. Como profilaxis antibiótica administramos una dosis de tobramicina $100 \mathrm{mg}$ endovenosa 30 minutos antes de la biopsia, seguida de ciprofloxacino $500 \mathrm{mg}$ cada 12 horas vía oral durante 72 horas.

Analizamos en todos los pacientes las siguientes variables: Edad, PSA, cociente PSA libre/total, PSA densidad, volumen prostático.

La estrategia de punción prostática consiste en la toma de los seis cilindros según la distribución sextante clásica (parauretrales craneal, medial, apical, derechos e izquierdos) a los que añadimos 4 cilindros de las zonas más laterales de la próstata (cuernos laterales) según el esquema de la Figura 1. Los cilindros son recogidos y procesados para el análisis anatomopatológico de forma separada.

Dado que la serie histórica data del año $2003^{14}$ y la distribución de las variables no era 


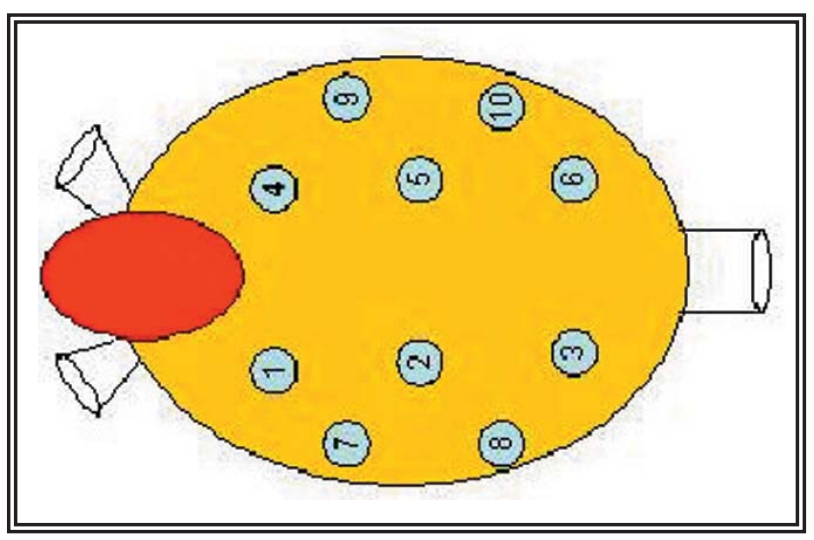

FIGURA 1: Esquema, distribución de las punciones prostáticas para pacientes que se someten a una primera biopsia prostática en el hospital universitario la Fe.

homogénea respecto de los pacientes que biopsiamos actualmente, desestimamos comparar los dos grupos, procediendo a analizar de forma prospectiva en cada paciente el rendimiento de los cilindros de la sextante, el de los 4 cilindros de los cuernos laterales y el de la biopsia ampliada con diez cilindros. Posteriormente comparamos la rentabilidad diagnóstica de cada zona entre ellas y con la ampliada.

Para el estudio estadístico de las variables continuas realizamos un análisis univariante comparativo con los test: T-student, para las variables cuantitativas; el test de McNemar, o de chi cuadrado, si seguían las variables eran cualitativas; el paquete informático empleado fue el SPSS $11.0{ }^{\circledR}$, los datos fueron recogidos en una base de datos de File Maker ${ }^{\circledR}$.
Tabla 1

Valores de las variables analizadas en cada paciente sometido a una primera biopsia prostática.

\begin{tabular}{lcccc}
\hline & Media & Rango & DE & IC 95\% \\
\hline EDAD & 63 & $51-78$ & 6,78 & $61,6-64,4$ \\
PSA & 6,73 & $4-10$ & 1,78 & $6,37-7,1$ \\
PSA 1/tot & 0,15 & $0,09-0,6$ & 0,077 & $0,132-0,165$ \\
VOL & 56,12 & $14-155$ & 30,1 & $49,96-62,29$ \\
PSA dens & 0,15 & $0,043-0,46$ & 0,08 & $0,133-0,166$ \\
\hline
\end{tabular}

adenocarcinoma fue detectado únicamente en los cilindros de los cuernos laterales, o lo que es lo mismo; cada 11 biopsias diagnosticamos un nuevo cáncer de próstata gracias a los cilindros de los cuernos laterales, número necesario para tratar (NNT): 11,36; o dicho de otro modo, cada 100 pacientes que biopsiamos a 9 les ahorramos una segunda biopsia.

Comparando ahora la rentabilidad de la biopsia sextante respecto a la ampliada a 10 cilindros se objetivan diferencias estadísticamente significativas (test de McNemar $\mathrm{p}=0.008$ ), observando una rentabilidad diagnóstica un $27 \%$ superior de la técnica de los 10 cilindros frente a la sextante clásica (Tabla 2).

No existen diferencias cuando comparamos la tasa diagnóstica de los cilindros de los cuernos laterales con la de la sextante clásica (Tabla 3).

Con el fin de valorar la relevancia clínica de los tumores "extra" diagnosticados en los cilindros de los cuernos laterales, hacemos un análi-

\section{RESULTADOS}

Los valores de las variables edad, PSA, PSA libre/total, volumen prostático, y PSA densidad se representan en la Tabla 1.

En la biopsia ampliada con toma de diez cilindros se detectan 37 pacientes con cáncer (41\%). De estos 37 tumores, 29 (el 78,37\%) hubieran sido diagnosticados con los cilindros de la sextante clásica.

Con los cilindros de los cuernos laterales exclusivamente, se hubieran diagnosticado 31 tumores (el 83,7\%) (Fig. 2). En 8 pacientes con cáncer de próstata, el

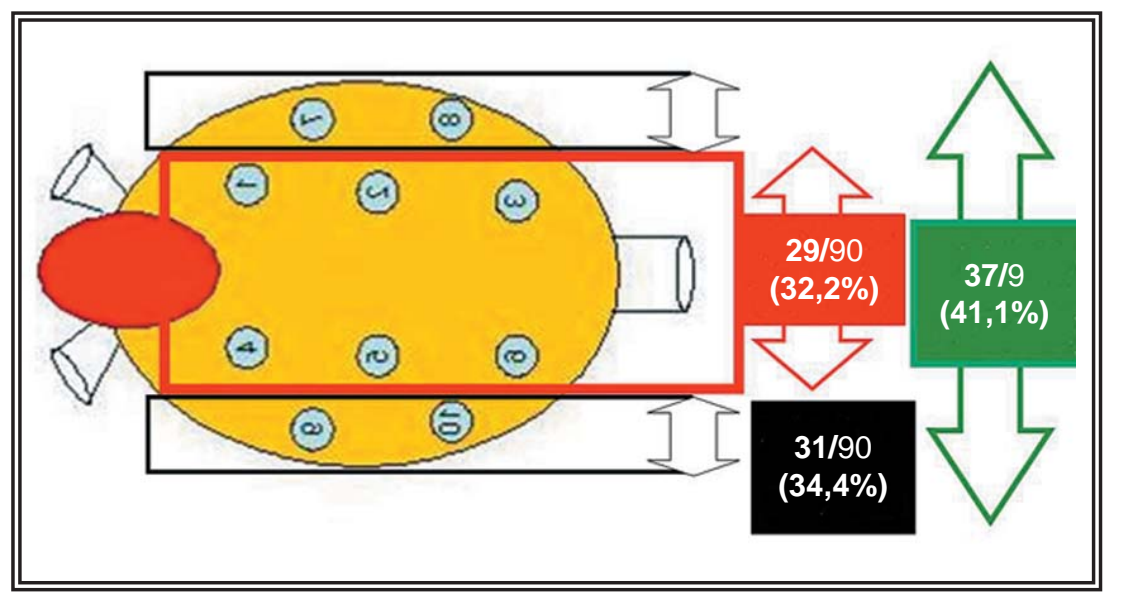

FIGURA 2: Representación gráfica de las rentabilidades diagnósticas de las distintas zonas biopsiadas: rentabilidad de los cilindros de la sextante clásica 32,2\%, de los cuernos laterales 34,4\%. Tasa diagnóstica global: 41,1\%. 
Tabla 2

Tabla 2x2 rentabilidades diagnósticas de las biopsias sextante frente a la ampliada (MacNemar test; $p=0,08$ ).

\begin{tabular}{lccc}
\hline Ampliada & & Sextante & Total \\
\hline+ & 29 & - & $\mathbf{3 7}(\mathbf{4 1 , 1 \% )}$ \\
- & 0 & 53 & 53 \\
Total & $\mathbf{2 9 ( 3 2 , 2 \% )}$ & 61 & $\mathbf{9 0}$ \\
\hline
\end{tabular}

Tabla 3

Tabla 2x2 rentabilidades diagnósticas de las biopsias sextante frente a la biopsia de los 4 cilindros de los cuernos laterales (MacNemar test; $p=0,78$ ).

\begin{tabular}{lccc}
\hline $\begin{array}{c}\text { Cuernos } \\
\text { Laterales }\end{array}$ & $\begin{array}{c}\text { Sextante } \\
+\end{array}$ & - & Total \\
\hline+ & 23 & 8 & $\mathbf{3 1}(\mathbf{3 4 , 4 \% )}$ \\
- & 6 & 53 & 59 \\
Total & $\mathbf{2 9 ( 3 2 , 2 \% )}$ & 61 & $\mathbf{9 0}$ \\
\hline
\end{tabular}

sis descriptivo de los ocho pacientes en cuestión (Tabla 4). La edad media es de 66 años; el PSA medio de 6,75 , a 4 de ellos se les detecta un adenocarcinoma prostático Gleason 7 o mayor. Otro paciente presenta un Gleason 6 y otros dos, grado 4 y 3 .

\section{DISCUSIÓN}

La sensibilidad de la biopsia prostática transrectal ecodirigida no es del $100 \%$. El hecho de que en la literatura haya descritas tasas de detección de cáncer para pacientes con biopsias de repetición -en los que persiste la sospecha de

\section{Tabla 4}

Características de los pacientes diagnosticados gracias a los cilindros de los cuernos laterales

\begin{tabular}{lccccc}
\hline Edad & PSA & coc PSA & Vol & PSA den & Gleason \\
\hline 61 & 8,04 & 0,14 & 30 & 0,265 & $(4+4) 8$ \\
66 & 6,45 & 0,28 & 100 & 0,065 & nd \\
73 & 4,6 & 0,11 & 56 & 0,083 & $(2+2) 4$ \\
58 & 6,15 & 0,065 & 14 & 0,43 & $(3+4) 7$ \\
70 & 6,17 & 0,19 & 69 & 0,089 & $(3+4) 7$ \\
73 & 8,4 & 0,13 & 94 & 0,09 & $(2+3) 5$ \\
58 & 4,8 & 0,1 & 38 & 0,13 & $(4+3) 7$ \\
69 & 9,4 & 0,08 & 47 & 0,2 & $(3+3) 6$ \\
\hline
\end{tabular}

tumor- de hasta el $37 \%{ }^{9}$, sugiere que la estrategia seguida en las primeras biopsias de esas series no era la idónea. También hay grupos que refieren un aumento de la tasa diagnóstica con el incremento del número de cilindros: con 10, 12 ó 13 cilindros aumenta la proporción de diagnósticos en un $25,5 \%, 22 \%$, y $35 \%$ respectivamente ${ }^{15}$, 17. No obstante, también hay estudios que hallan resultados contrarios. ${ }^{18,19}$.

La mayoría de los esquemas propuestos para la biopsia prostática ampliada derivan de la sextante clásica. Al analizar el aumento de la tasa diagnóstica con las diferentes estrategias de biopsias ampliadas, dicho incremento viene dado por los cilindros más lateralizados ${ }^{20-22}$. Así, por ejemplo, Eskew et al en un estudio donde por primera vez se compara una estrategia con toma de 13 biopsias a la sextante, objetivaron una reducción del 35\% en los falsos negativos; un $88 \%$ de los tumores que se diagnosticaron con los 5 cilindros añadidos a la sextante fueron localizados en los cuernos laterales ${ }^{21}$. También Presti et $\mathrm{al}^{20}$ que biopsian a 483 pacientes con una técnica semejante a la nuestra, consiguen una sensibilidad del 96\%, y además plantean que la retirada del cilindro de la zona periférica craneal de la sextante de ambos lóbulos sólo supondría dejar de diagnosticar un $1 \%$ de los tumores ${ }^{20}$. En este estudio la sextante clásica no diagnosticó un $20 \%$ de los tumores ${ }^{20}$. En nuestra serie no tenemos suficiente potencia estadística para encontrar diferencias entre las tasas diagnósticas de cada uno de los cilindros que tomamos, por lo que no nos planteamos si se podría prescindir de alguno de ellos.

Desestimamos la punción de la zona transicional pues la literatura señala que aunque un $20 \%$ de los tumores se originan dicha localización, el incluir en las primeras biopsias un cilindro de ésta zona, incrementa muy poco la rentabilidad diagnóstica $(1,8 \%-4,3 \%)^{23}, 24$. Sin embargo en las segundas biopsias la rentabilidad aumenta hasta un 10\%-13\%25, 26. Lui et $\mathrm{al}^{26}$ encuentran un 53\% de tumores en la zona transicional de segundas biopsias de pacientes con un PSA medio de $32 \mathrm{ng} / \mathrm{ml}$ y tacto rectal normal.

En la mayoría de los estudios publicados, las series sobre las que se realizan estos trabajos no 
son homogéneas. Por ello en el presente trabajo incluimos a los pacientes que acuden para una primera biopsia, y con un PSA entre 4 y 10, con el fin de seleccionar aquellos con mayor probabilidad de presentar enfermedad organoconfinada. Además seleccionamos los que tienen un tacto rectal no sospechoso puesto que la frecuencia de biopsia positiva para pacientes con tacto sospechosa alcanza el $56 \% \%^{27}$. En la mayoría de las series publicadas existe una proporción de pacientes con tacto rectal sospechoso que oscila entre el $18 \%$ de la serie de Babaian ${ }^{28}$ y el $52 \%$ de la de $E_{s k e w^{21}}$, en otras ni siquiera se precisa ${ }^{20}$. Babaian $^{28}$ si que estratifica sus resultados en función del tacto rectal y cuando es normal la tasa de detección de su modalidad de biopsia ampliada es del $16 \%$.

Pese a que el rango de edad de nuestros pacientes oscila entre los 51 y 78 años ya que no biopsiamos a pacientes con una esperanza de vida menor de 10 años, en el estudio univariante encontramos una asociación estadística entre edad y cáncer. Al igual que los estudios de la mayoría de los autores ${ }^{18,19,28}$ objetivamos una asociación estadística entre la aparición de cáncer y volúmenes prostáticos pequeños. Y muy en relación con este hecho también encontramos asociación significativa entre las altas densidades de PSA y el cáncer. Probablemente por la falta de potencia estadística y el estrecho rango de los valores de PSA total no encontremos asociación entre el PSA total y el cociente PSA libre/total, con la aparición de cilindros positivos en la biopsia.

El mero hecho de lateralizar las punciones de las biopsias en sextante aumenta un 23\% la tasa de diagnóstico de cáncer ${ }^{29}$, 30 , quizás sea esa la razón por la que Rodríguez Patrón et al obtuvieran altas tasas de diagnóstico con toma de seis cilindros y no encuentrarán diferencias en la proporción de tumores cuando amplían el número de tomas ${ }^{19}$.

Las características de nuestros 90 pacientes estudiados no son superponibles a la de las demás series publicadas pues contamos con un mayor volumen prostático que las series anglosajonas, un PSA medio menor, además de que la mayoría incluyen pacientes con tacto rectal sugestivo de tumor.
Numerosos estudios indican que el aumento del número de cilindros incrementa la tasa de detección de cáncer ${ }^{20}, 21,28,30$. Sin embargo Naughton ${ }^{18}$ y Rodríguez-Patrón ${ }^{19}$ no encuentran mayor rentabilidad con las biopsias ampliadas. $\mathrm{El}$ primero no encuentra diferencias significativas al comparar la sextante clásica con la toma de 12 cilindros, pero cuando analiza el grupo de biopsia ampliada objetiva que los cilindros "extra" detec$\tan 7$ tumores que hubiesen pasado inadvertidos con la sextante, lo que supone aumentar un $21 \%$ el rendimiento de la sextante. Posiblemente, como el propio grupo de Rodríguez-Patrón explica, no encontraron diferencias porque en los pacientes en el que realizaban la sextante el urólogo instintivamente lateralizaba la entrada de la aguja de resorte, detectando con ello más tumores. De hecho, desde 1994, el grupo de la universidad de Alcalá de Henares realiza una técnica en sextante lo más lateralizada posible ${ }^{29}$.

En nuestra serie vemos que la tasa global de detección de cáncer es del 41,1\%. Existen diferencias estadísticamente significativas cuando la comparamos la rentabilidad global con el porcentaje de tumores detectados exclusivamente por los cilindros de la sextante. Y lo que es más, con los 4 cilindros de los cuernos laterales se detectaron dos tumores más que con los 6 de la sextante, si bien estas diferencias no son estadísticamente significativas. La sextante, por sí sola, hubiese obviado 8 tumores de próstata, 4 de ellos con un Gleason 7 u 8 en pacientes con una edad de $61,58,70$, y 58 años.

\section{CONCLUSIONES}

La constatación en piezas de prostatectomía radical de una alta presencia de adenocarcinomas de próstata en los cuernos laterales ${ }^{30}, 31$, sugiere la conveniencia de incluir esa área en la toma de biopsias. Por ello consideramos que la biopsia ampliada a 10 cilindros con la distribución de la Figura 1, es una estrategia adecuada para los pacientes con sospecha de adenocarcinoma prostático que van a ser sometidos a su primera biopsia. Teniendo en cuenta la relevancia clínica de los tumores diagnosticados gracias a los cilindros "extra", no pensamos que esta técnica conlleve un sobrediagnóstico del cáncer de próstata, y con ello sobretratamiento. 


\section{REFERENCIAS}

1. Lee F, Gray JM, McLeary RD, Meadows TR, Kumasaka GH, Borlaza GS, et al. Transrectal ultrasound in the diagnosis of prostate cancer: localization, echogenicity, histopatology, and staging. Prostate. 1985;7(2):117-129.

2. Torp Pedersen LF, LeeF, Siders DB. The role of transrectal ultrasound in the early detection of prostate cancer. CA Cancer J Clin. 1989;39(6):337-360.

3. Radge H, Aldape HC, Bagley CM Jr. Ultrasound guided prostate biopsy: biopsy gun superior to aspiration. Urology. 1988; 32(6):503-506.

4. Hodge KK, Macneal JE, Stamey TA. Ultrasound guided transrectal core biopsy of the palpably abnormal prostate. J Urol. 1989;142(1):66-70.

5. Pelzer A, Bektic J, Berger AP, Pallwein L, Halpern EJ, Horninger $\mathrm{W}$ et al. Prostate cancer detection in men with prostate specific antigen 4 to $10 \mathrm{ng} / \mathrm{ml}$ using a combined approach of contrast enhanced color Doppler targeted and systematic biopsy. J Urol. 2005;173(6):1926-1929.

6. Frauscher F, Pallwein L, Klauser A, Berger AP, Koppelstaetter F, Gradl $\mathrm{J}$ et al. Ultrasound contrast agents and prostate cancer Radiologe. 2005;45(6):544-551.

7. Mehta SS, Azzouzi AR, Hamdy FC. Three dimensional ultrasound and prostate cancer. World J Urol. 2004;22(5): 339-345.

8. Ito H, Kamoi K, Yokoyama K, Yamada K, Nishimura T. Visualization of prostate cancer using dynamic contrastenhanced MRI: comparison with transrectal power Doppler ultrasound. Br J Radiol. 2003;76(909):617-624.

9. Yuen JS. Re: Comparison of contrast enhanced color Doppler targeted biopsy with conventional systematic biopsy: impact on prostate cancer detection. J Urol. 2003; 170(1):192-193.

10. Ellis WJ, Chetner MP, Preston SD, Brawer MK. Diagnosis of prostatic carcinoma: The yield of serum prostate specific antigen, digital rectal examination and transrectal ultrasonography. J Urol. 1994;152(5 Pt1):1520-1525.

11. Hammerer PG. Sonographic diagnosis of prostatic cancer. Radiologe. 1994;34(3):116-121.

12. Hammerer PG, Huland H. Systematic sextant biopsies in 651 patients referred for prostate evaluation. J Urol. 1994;151(1):99-102.

13. Carter HB, Hamper UM, Sheth S, Sanders RC, Epstein JI, Walsh PC. Evaluation of ultrasound in the early detection of prostate cancer. J Urol. 1989;142(4):1008-1010.

14. Gómez Ferrer A: Análisis computerizado de la imagen computerizada transrectal en el cáncer de próstata localizado. 2004 Tesis Doctoral Univ. Valencia.

15. Nash PA, Bruce JE, Indudhara R, Shinohara K. Transrectal ultrasound guided prostatic nerve blockade eases systematic needle biopsy of the prostate. J Urol. 1996; 155(2):607-609.

16. Eskicorapci SY, Bayda DE, Akbal C, Sofikerim M, Gunay M, Ekici S et al. An extended 10-core transrectal ultrasonography-guided prostate biopsy protocol improves the detection of prostate cancer. Eur Urol. 2004;45(4):444448.

17. Beurton D, Barthelemy Y, Fontaine E. Twelve systematic prostate biopsies are superior to sextant biopsies for diagnosing carcinoma: a prospective randomized study. $\mathrm{Br} \mathrm{J}$ Urol. 1997;80(2):239-242.
18. Naughton CK, Miller DC, Mager DE, Ornstein DK, Catalona WJ. A prospective randomized trial comparing 6 versus 12 prostate biopsy cores: impact on cancer detection. J Urol. 2000;164(2):388-392.

19. Rodriguez-Patron Rodriguez R, Mayayo Dehesa T, Alonso Gonzalez M, Burgos Revilla FJ, Garcia Gonzalez R, Lennie Zucharino A. Rentabilidad de la biopsia de prostate ampliada con 10muestras prostáticas. Estudio prospectivo sobre 222 pacientes. Arch Esp Urol. 2006;59(1):15-24.

20. Presti, JC Jr, Chang JJ, Bhargava V, Shinohara K. The optimal systematic prostate biopsy scheme should include 8 rather than 6 biopsies: results of a prospective clinical trial. J Urol. 2003;163(1): 163-170.

21. Eskew LA, Bare RL, McCullough DL. Systematic 5 region prostate biopsy is superior to sextant method for diagnosing carcinoma of the prostate. J Urol. 1997;157(1):199-202.

22. Singh H, Canto EI, Shariat SF, Kadmon D, Miles BJ, Wheeler TM et al. Improved detection of clinically significant, curable prostate cancer with systematic 12-core biopsy. J Urol. 2004;171(3):1089-1092.

23. Bazinet M, Karakiewicz PI, Aprikian AG, Trudel C, Aronson $\mathrm{S}$, Nachabe $\mathrm{M}$ et al. Value of systematic transition zone biopsies in the early detection of prostate cancer. J Urol. 1996;155(2):605-606.

24. Epstein JI, Walsh PC, Sauvegeot J, Carter HB. Use of repeat sextant and transition zone biopsies for assessing extent of prostate cancer. J Urol. 1997;158(5):1886-1890.

25. Keetch DW, Catalona WJ. Prostatic transition zone biopsies in men with previous negative biopsies and persistently elevated serum prostatic specific antigen values. J Urol. 1995;154(5):1795-1797.

26. Lui PD, Terris MK, McNeal JE, Stamey TA. Indications for ultrasound guided transition zone biopsies in the detection of prostate cancer. J Urol. 1995;153(3):1000-1003.

27. Ravery V, Goldblatt L, Royer B. Extensive biopsy protocol improves the detection rate of prostate cancer. J Urol. 2000; 164(2):393-396.

28. Babaian RJ, Toi A, Kamoi K, Troncoso P, Sweet J, Evans R. A comparative analysis of sextant and an extended 11 core multisite directed biopsy strategy. J Urol. 2000; 163 (1):152-157

29. Rodriguez-Patrón Rodriguez R, Mayayo Dehesa T, Burgos Revilla FJ, Alonso Gonzalez M, Lennie Zucharino A, Garcia Gonzalez R. Resultados de una serie de 6000 pacientes con biopsia transrectal ecodirigida de próstata. Parte I; Anatomía Patológica, Tacto rectal, Ecografía transrectal y PSA. Arch Esp Urol. 2005;58(7):623-634.

30. Bauer JJ, Zeng J, Weir J, Zhane W, Sesterhenn IA, Connelly RR et al: Three-dimensional computer-simulated prostate models: lateral prostate biopsies increase the detection rate of prostate cancer. Urology 1999(5);53:961-967.

31. Mazal PR, Haitel A, Windischberger C, Djavan B, Sedivy R, Moser E et al. Spatial distribution of the prostate cancers undetected on initial needle biopsies. Eur Urol. 2001; 39(6):662-665.

Correspondencia autor: Dr. M. Ramírez Backhaus Servicio de Urología. Hospital Universitario La Fe Avda. Campanar 21, 46009 Valencia. Tel.: 963862700 E-mail autor: ramirezbackhaus@yahoo.es Información artículo: Original - Cáncer de próstata (Trabajo aceptado noviembre 2006) 